\title{
Archéopages
}

Archéopages

Archéologie et société

Hors-série 1 | 2008

Constructions de l'archéologie

\section{L'archéologie paléolithique à la reconquête de territoires oubliés}

Marc Jarry

\section{(2) OpenEdition}

1 Journals

Édition électronique

URL : https://journals.openedition.org/archeopages/838

DOI : 10.4000/archeopages.838

ISSN : 2269-9872

Éditeur

INRAP - Institut national de recherches archéologiques préventives

Édition imprimée

Date de publication : 1 février 2008

Pagination : 28-33

ISSN : $1622-8545$

\section{Référence électronique}

Marc Jarry, "L'archéologie paléolithique à la reconquête de territoires oubliés », Archéopages [En ligne], Hors-série 1 | 2008, mis en ligne le 01 février 2008, consulté le 24 février 2023. URL : http:// journals.openedition.org/archeopages/838 ; DOI : https://doi.org/10.4000/archeopages.838

Tous droits réservés 


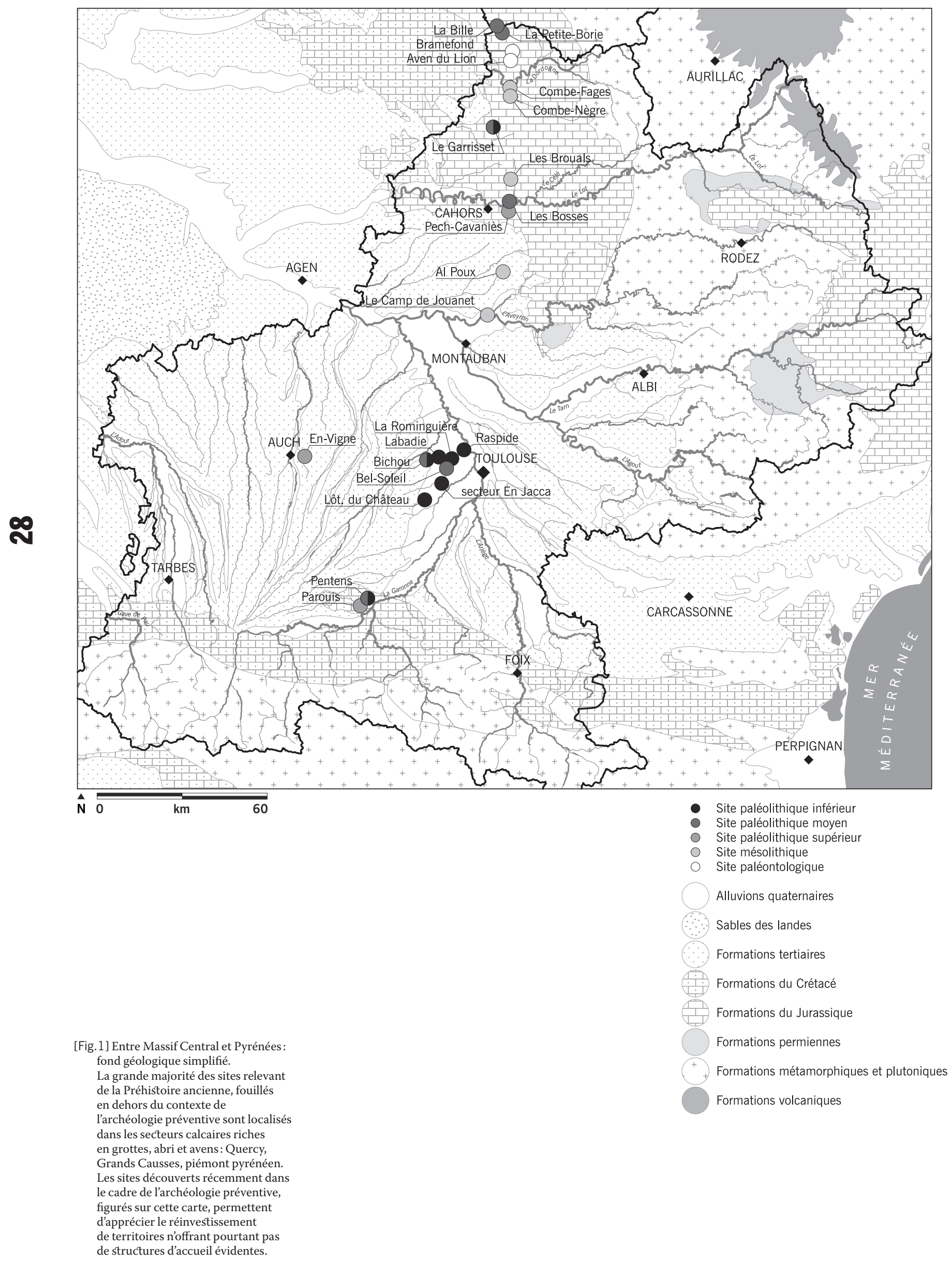




\section{L'archéologie paléolithique à la reconquête de territoires oubliés}

\author{
Marc Jarry \\ Inrap, UMR 5608, TRACES-UTAH
}

L e mythe de la caverne préhistorique. Plonger dans les profondeurs de l'histoire de l'humanité n'est pas une mince affaire. Il y a toujours eu, pour les disciplines se consacrant aux phases anciennes de la Préhistoire, un lien implicite avec l'éternelle quête des origines. L'archéologie s'est alors trouvée confrontée à des explications légendaires issues de concepts spirituels fermement ancrés au cœur des cultures, notamment pour celles enracinées dans les religions du Livre. L'étude scientifique des civilisations anciennes devait passer préalablement par la démonstration de l'action humaine sur les vestiges retrouvés, puis de leur ancienneté. Les mythes originels exégétiques ne sont pourtant pas encore totalement écartés des conceptions de certains de nos contemporains, partisans d'une cosmogonie anthropocentrique au dessein immuable. Toutefois, il faut convenir que l'archéologie préhistorique a réussi à faire admettre au plus grand nombre la réalité de sa propre genèse, même s'il nous reste encore à extraire de l'imaginaire collectif la figure forcée de l'homme-singe, véritable exutoire de notre impensable animalité.

Néanmoins, il faut avouer que, au-delà des barrières spirituelles et psychologiques qu'il a fallu franchir, les vestiges des humanités antédiluviennes ne sont pas aussi évidents à déchiffrer que ceux des civilisations plus proches de la nôtre. Il n'est pas facile, de prime abord, d'admettre l'intervention de l'homme dans les «pierres de foudre» de Lucrèce. Sans intromission éclairée, il n'est pas aisé de percevoir la main de l'artisan préhistorique, fort de sa maîtrise technique et de son savoir-faire ancestral, dans le ballast siliceux d'un amas de débitage. Il faut reconnaître que l'archéologie des phases anciennes de la Préhistoire n'est que très rarement spectaculaire. Bien sûr, les expressions artistiques en constituent les manifestations les plus indéniables et surprenantes, de même que les fossiles humains nous interpellent comme autant de vanités, mais le reste n'est pas vraiment sensationnel. Point d'or ni de trésor! Et plus on s'enfonce dans les temps immémoriaux, plus cela est flagrant. La Préhistoire ancienne, si elle n'est pas spectaculaire ou exotique, doit donc, après avoir vaincu les incrédulités, persuader de sa valeur intrinsèque, de son utilité pour la connaissance.

Mais ce n'est malheureusement pas tout: non seulement les découvertes ne sont pas souvent prestigieuses (sensu rentables), mais, qui plus est, elles restent rares, mal conservées, difficiles à repérer et délicates à exploiter. Il n'y a donc rien d'étonnant à ce que la recherche se soit concentrée presque exclusivement dans des secteurs recelant des grottes et des abris. En effet, ces pièges sédimentaires, assez clairement perceptibles dans les paysages, offrent un important potentiel informatif et donc de fortes chances de retour sur investissement. Entre autres avantages, la stratigraphie y est souvent assez développée, permettant l'approche diachronique; les vestiges et notamment la faune y sont généralement bien préservés, apportant des données fondamentales sur les moyens de subsistance des groupes ou encore la saisonnalité d'occupation. Mais, en dehors des régions karstiques, la recherche s'est de fait limitée aux découvertes fortuites ou aux prospections de surface, toujours difficiles à appréhender et de toute façon révélatrices de gisements déjà détruits ou bien entamés. Le préhistorien, scrutant les ombres du passé, a alors bien malgré lui des difficultés à regarder hors de la grotte...

Entre Massif central et Pyrénées... Mais où fouiller? Comment repérer un site en n'ayant aucun point de repère? Où implanter un sondage? En plein air, les sites sont indétectables, à quelques rares exceptions près. Les chances de tomber sur un gisement sont infimes. Ce n'est qu'avec le plein développement de l'archéologie préventive de ces vingt dernières années et l'acuité même de sa méthode d'intervention par sondages systématiques «en aveugle» que les investigations ont pu enfin reconquérir des terrains jusqu'alors quelque peu oubliés.

En dehors de quelques péjorations climatiques majeures, ayant provoqué des désertions épisodiques de certains secteurs, il est envisageable que la majeure partie du territoire français soit restée occupée durant toute la Préhistoire. Pour certaines régions, cela ouvre de belles perspectives de recherche qui n'ont, semble-t-il, été qu'effleurées pour l'instant. Dans le Sud-Ouest, fertile en découvertes récentes, l'espace compris entre le Massif central et la chaîne pyrénéenne est une bonne illustration de ce retour vers des espaces en jachère. La zone concernée est immense, englobant des contextes géologiques diversifiés, induisant des paysages contrastés, mais complémentaires [Fig.1]. La grande plaine alluviale de la Garonne, avec ses larges paliers de terrasses étagées, est une unité morphologique structurant cet ensemble. Ce puissant fleuve, au bassin ouvert sur l'Aquitaine, draine les deux massifs primaires qui l'encadrent, eux-mêmes bordés par des piémonts calcaires. D'un point de vue archéologique, cette région est très riche et le patrimoine préhistorique est à l'échelle de son ampleur géographique. En résumé, les sites majeurs, toutes périodes confondues, sont localisés dans les régions karstiques (Quercy, Grands Causses et piémont pyrénéen) et sont tous, à peu de chose près, associés à des grottes, des abris ou des avens. Les sites de plein air fouillés sont rarissimes. Pourtant, la carte archéologique est constellée 


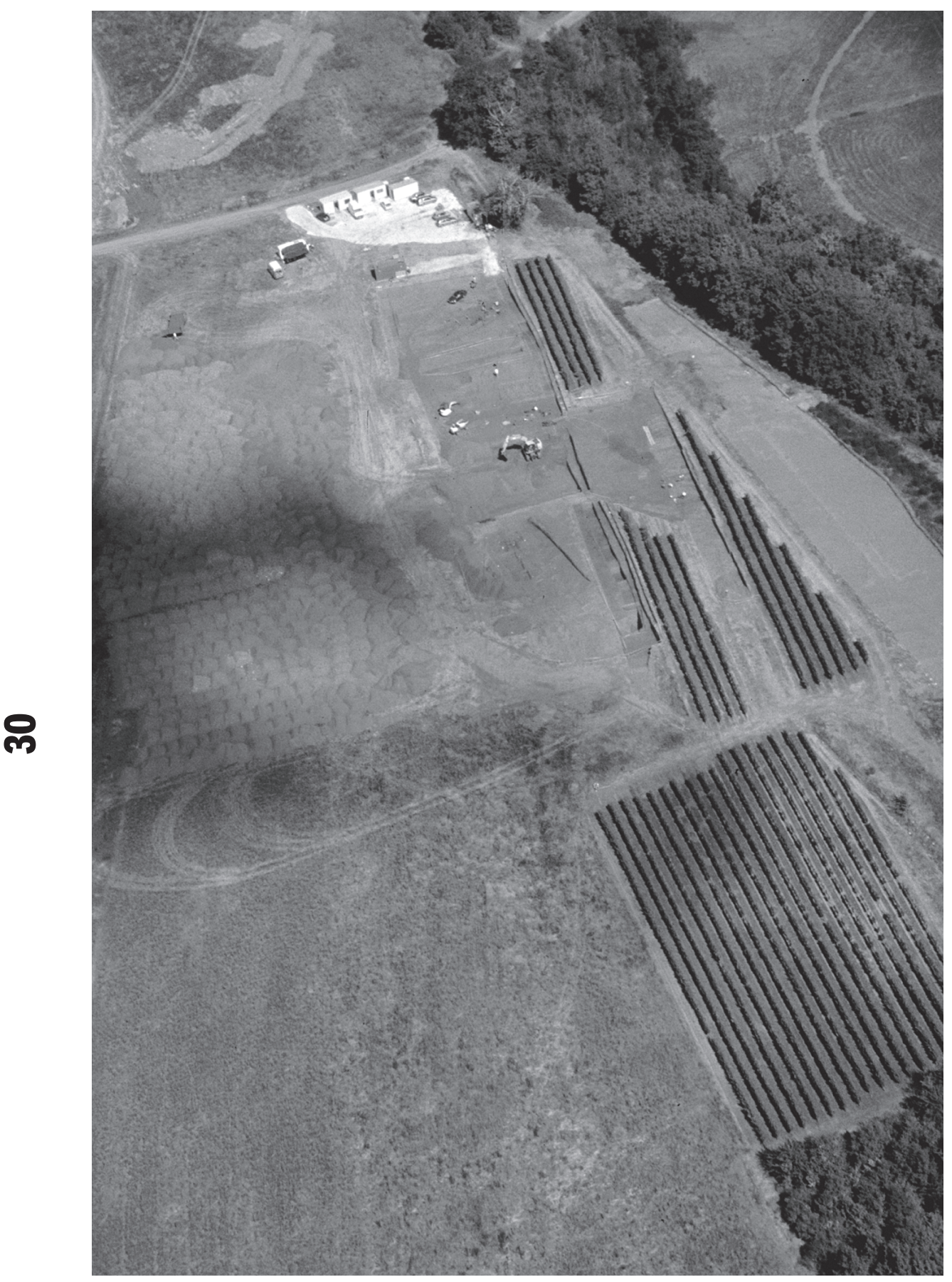

[Fig.2] Le gisement des Bosses

à Lamagdelaine (Lot) est un exemple

de l'apport significatif

de l'archéologie préventive

à la connaissance des manifestations

les plus anciennes du Paléolithique

moyen. Fouillé en 2000 dans

le cadre de la construction de

l'autoroute A2o et daté d'environ

300000 ans avant notre ère,

il est implanté sur une terrasse

alluviale du Lot et est

complémentaire des nombreux

gisements quercynois localisés

en contexte karstique . 
d'une myriade de points de ramassages en surface, témoins d'une occupation dense par les populations préhistoriques. Au mieux, des séries ont pu être récoltées in situ, au hasard de constructions de routes, d'exploitations d'argile ou du creusement de canaux. Ce phénomène est encore plus flagrant pour le Paléolithique inférieur du Midi toulousain. Les vestiges lithiques récoltés en surface sur les terrasses de la Garonne sont si nombreux que, à un moment donné, on a évoqué l'hypothèse d'un immense et inextricable gisement continu. Malgré cette abondante documentation, l'absence de contexte stratigraphique fiable constitue un véritable écueil.

Depuis les années 1990, l'archéologie préventive a connu quelques succès, notamment pour les Paléolithiques inférieur et moyen, permettant une véritable reconquête de territoires quelque peu délaissés. De nombreux sites, plus ou moins importants, ont pu être mis au jour, dans des lieux et à des niveaux de conservation insoupçonnés [Fig.2]. Le Paléolithique supérieur reste curieusement assez discret, mais il est encore difficile de dire si cette absence, notamment dans le Midi toulousain, est le reflet de la réalité ou simplement un artéfact qui persiste.

Au-delà de la reconquête physique d'un secteur géographique, qui ne ferait qu'alimenter la carte archéologique déjà bien fournie, les territoires recouvrés permettent d'investir des terrains de recherche nouveaux. En premier lieu, il est indéniable que les approches environnementales bénéficient grandement de l'exploration systématique de vastes espaces. Un autre corollaire de cette expansion territoriale est l'abord de terroirs aux ressources lithologiques diversifiées. Par exemple, les corps alluviaux garonnais dans le Midi toulousain contiennent un cortège lithologique très varié, où dominent les quartzites, les quartz, les lydiennes ou encore les schistes. Les silex sont rares, voire très rares. Logiquement, les artisans préhistoriques ont largement puisé leurs matières premières au sein de ces ressources, notamment pendant les phases anciennes du Paléolithique où la mobilité des groupes apparaît moindre [Fig.3]. Les problématiques de recherche évoluent ainsi, réinvestissant ces domaines spécifiques de la pétroarchéologique et des études technoéconomiques. Certes, les industries ne sont pas toujours spectaculaires, mais elles sont tout autant les vestiges des activités passées. Ces matériaux mis en œuvre par les artisans préhistoriques apparaissent par ailleurs particulièrement adaptés à leurs besoins.

\section{Le préhistorien des cavernes réinvestit les territoires des Préhistoriques.}

Les investigations dans les structures d'accueil hypogées, de très haute qualité au demeurant, se trouvent donc privées d'une part véritablement conséquente de la documentation provenant de l'extérieur. En outre, cette agrégation des chercheurs autour des cavités a contribué à la propagation de l'image caricaturale et en partie fausse de «l'homme des cavernes». Les découvertes fortuites apportaient bien un tribut à la science; et Boucher de Perthes, dès 1840 dans les carrières de la vallée de la Somme, est le premier à les mettre à profit pour apporter la preuve de l'ancienneté des industries en pierre taillée. De même, les prospections de surface autorisent une cartographie des implantations humaines, levant quelque peu le voile sur le potentiel du plein air. Mais, en France, ce n'est qu'avec les premières grandes fouilles «de sauvetage » effectuées dans les années 1980 qu'apparaît de manière patente l'amplitude du champ des possibles pour la connaissance de la Préhistoire ancienne. L'archéologie préventive, par les méthodes qui lui sont propres, explore maintenant de manière systématisée l'ensemble des territoires géographiques, ouvrant des perspectives vers de nouveaux travaux. On retrouve alors une notion essentielle en archéologie préhistorique: le(s) territoire(s). En effet, les populations de chasseurs-collecteurs, par définition mobiles, occupent des espaces plus ou moins vastes qu'elles s'approprient et investissent au fil des saisons. Cet ancrage géographique des groupes préhistoriques dans les paysages doit pouvoir être appréhendé dans toute son extension, ses modalités d'occupation et d'exploitation dans des environnements diversifiés où les sites apparaissent comme complémentaires.

Nous ne sommes encore qu'aux prémisses de la perception de la réalité des territoires préhistoriques. L'archéologie préventive apporte sa pierre à l'édifice, mais la lumière sur le plein air n'est pas une quête tranquille. Elle doit encore se réapproprier des secteurs géographiques. L'intégralité du pays ne bénéficie pas toujours du même regain d'activité, signe que l'effort doit être poursuivi. Il faut encore convaincre, former, recruter, pérenniser. La découverte des sites de plein air reste très difficile et aléatoire. Elle exige beaucoup de patience, d'expérience, de moyens. Reconnaître un site préhistorique sous le godet d'une pelle mécanique est loin d'être évident: les objets lithiques apparaissent ton sur ton, très fugaces, invisibles à l'œil non averti. En outre, les phénomènes taphonomiques sont très destructeurs, occultant sans doute la part réelle de l'emprise humaine...

Mais percevoir la Préhistoire ancienne, c'est l'appréhender dans sa globalité anthropologique et environnementale. Tout concourt à la connaissance : les vestiges prestigieux ou non, en grotte ou en plein air, en silex ou en quartz, même les sondages négatifs sont porteurs d'informations précieuses. L'archéologie préventive, par le fait qu'elle ne programme pas, au sens où elle ne choisit pas son territoire physique d'investigation, autorise l'acquisition d'une documentation inédite et rafraîchissante sur les sites de plein air. Sauf à considérer que la grotte fut le lieu de vie exclusif des Préhistoriques, on admettra facilement le caractère opportun de pouvoir disposer 

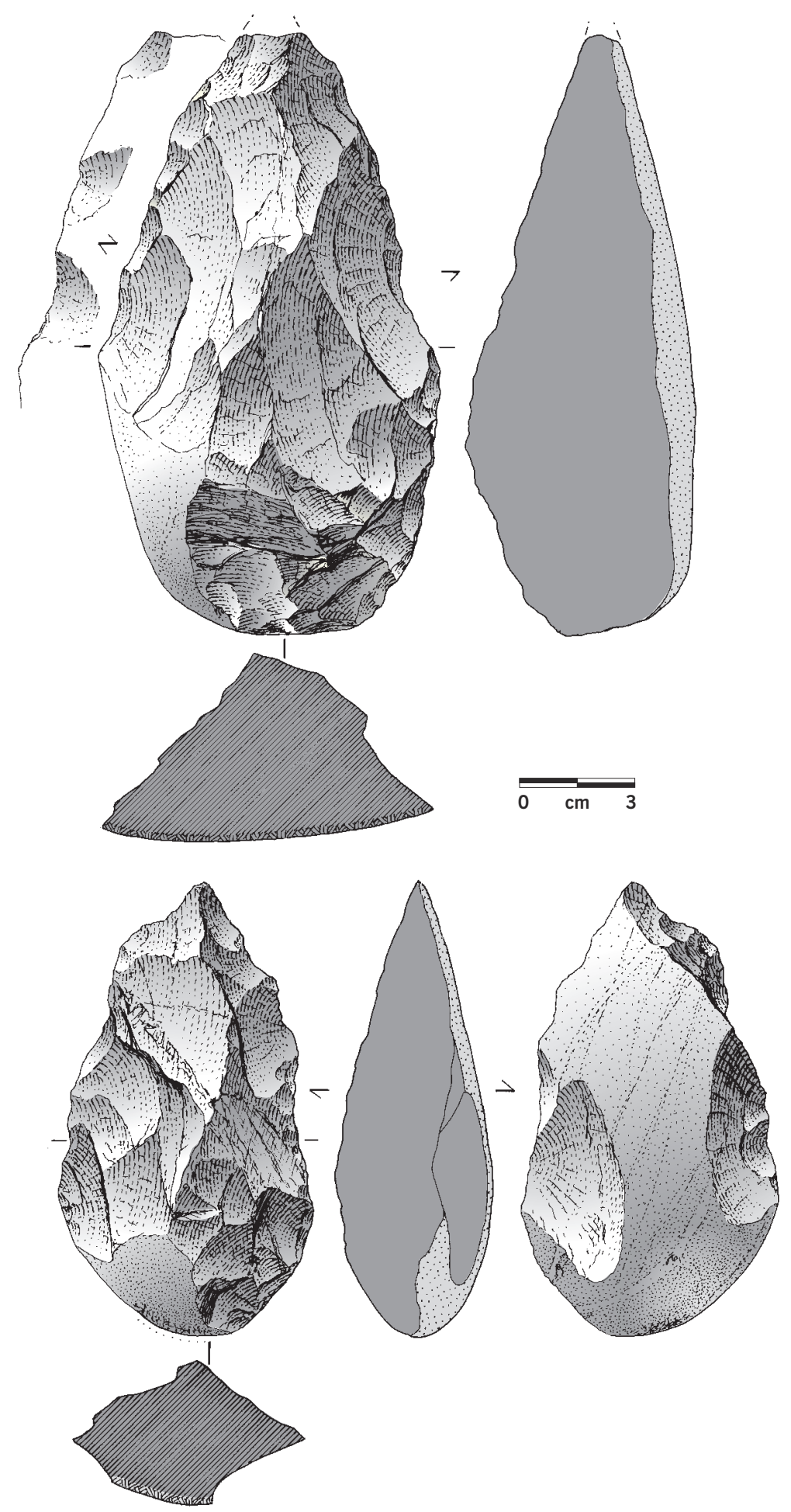

[Fig.3] Très majoritairement constituées de quartzites, les industries lithiques paléolithiques découvertes dans

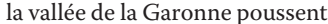

à se pencher sur l'étude des territoires archéopétrographiques quelque peu délaissés, faute de séries homogènes

jusqu'à présent (site de Bichou à Montaigut-sur-Save en HauteGaronne). 